\title{
Reconstruction of the ocular surface by autologous transplantation of rabbit adipose tissue-derived stem cells on amniotic membrane
}

\author{
Ting Qiu ${ }^{1}$, Lei Cui ${ }^{2}$, Jian-Jiang Xu ${ }^{3}$, Jia-Xu Hong ${ }^{3}$, Jun Xiang ${ }^{3}$ \\ ${ }^{1}$ Department of Ophthalmology, Shanghai Children's Medical Center, Shanghai Jiao Tong University School of Medicine, Shanghai, China; \\ ${ }^{2}$ National Engineering Research Center for Tissue Engineering, Shanghai, China; ${ }^{3}$ Department of Ophthalmology, Eye, Ear, Nose, and Throat \\ Hospital of Fudan University, Shanghai, China \\ Contributions: (I) Conception and design: JJ Xu; (II) Administrative support: L Cui; (III) Provision of study materials or patients: L Cui; (IV) \\ Collection and assembly of data: T Qiu; (V) Data analysis and interpretation: T Qiu; (VI) Manuscript writing: All authors; (VII) Final approval of \\ manuscript: All authors. \\ Correspondence to: Jian-Jiang Xu. Department of Ophthalmology, Eye, Ear, Nose, and Throat Hospital of Fudan University, Shanghai, China. \\ Email: jianjiangxu@126.com.
}

Background: Corneal disease is the second most common cause of blindness in China. Clinically, treatment options for corneal diseases with limbal stem cell deficiency (LSCD) are limited due to a shortage of organ donors and inevitable immune rejection. This study aims to determine the efficacy of reconstructing the ocular surface using autologous cultivated adipose tissue-derived stem cells (ADSCs) and to develop a new clinical treatment for patients with LSCD.

Methods: A rabbit LSCD model was first established. Two weeks later, the animals were divided into three groups, including the sham group, the amniotic membrane transplantation group, and the ADSC combined with amniotic membrane transplantation group, and underwent surgery. The efficacy of reconstructing the ocular surface using ADSCs was evaluated using immunofluorescent staining, confocal microscopy (CM) observation, H\&E staining, immunohistochemical staining, and scanning transmission electron microscopy observation one, two and four weeks after surgery.

Results: Evaluations of immunofluorescent staining of the cornea pre- and post-surgery yielded significantly lower scores for the corneas in the ADSCs transplantation group than for those in the sham group $(\mathrm{F}=-7, \mathrm{P}=0.002,<0.05)$ and the amniotic membrane transplantation group $(\mathrm{F}=-4.67, \mathrm{P}=0.018,<0.05)$ two weeks after surgery. Four weeks after surgery, the corneas of the ADSC combined with amniotic membrane transplantation group were scored significantly lower than those in the sham group $(\mathrm{F}=-8$, $\mathrm{P}=0.007,<0.05)$ and the amniotic membrane transplantation group $(\mathrm{F}=-5.33, \mathrm{P}=0.046,<0.05)$. The data suggest that the use of ADSCs to treat LSCD showed greater efficacy than the other treatment methods. The growth of ADSCs on the corneal surface was examined using confocal and electron microscopes. K3/ K12 expression in the corneal epithelium, which was reconstructed by ADSCs, was negative, as shown by immunohistochemical staining.

Conclusions: Ocular surface reconstruction can be improved by using ADSCs as seed cells and the amniotic membrane as a carrier, thus providing a new therapeutic strategy for patients with LSCD.

Keywords: Tissue engineering; limbal stem cell deficiency (LSCD); corneal epithelium, adipose tissue-derived stem cells (ADSCs); autologous transplantation

Submitted Apr 25, 2020. Accepted for publication Jul 22, 2020.

doi: $10.21037 / \mathrm{atm}-20-4368$

View this article at: http://dx.doi.org/10.21037/atm-20-4368 


\section{Introduction}

Corneal disease is one of the most common eye diseases in China that can lead to vision loss or blindness. Corneal diseases are the second-leading cause of blindness; only cataracts are a greater cause (1). Clinically, many severe impairments of the ocular surface, such as severe trauma (chemical injury, thermal burns, etc.), Stevens-Johnson syndrome, and ocular cicatricial pemphigoid, are all related to limbal stem cell deficiency (LSCD). Eventually, these diseases lead to blindness due to conjunctival epithelialization and neovascularization in the cornea because the cornea epithelium loses its ability to regenerate and repair. Clinically, treatment options for corneal diseases with LSCD are scarce due to a shortage of organ donors and inevitable immune rejection.

Adipose-derived stem cells (ADSCs) are a type of multipotent adult stem cell isolated from adipose tissue. They are similar to bone marrow mesenchymal stem cells in terms of biological properties, phenotypes, and multi-directional differentiation potentials. Featured by rich sources, easy sampling, little damage to the body, rapid proliferation in vitro, stable biological characters, high survival rate after grafting, and good environmental adaption and constant high-quality differentiation after implantation, ADSCs are ideal seed cells for tissue engineering and can be used as a potentially major source of human stem cell banks.

Current research has focused on tissue engineering of the corneal epithelium. In the present study, for the first time, we extracted adipose tissue-derived stem cells (ADSCs) from subcutaneous adipose tissue on the back of a rabbit's neck and validated their use (2). We have preliminarily demonstrated that ADSCs have multi-directional differentiation potential and can grow on the amniotic membrane ex vivo. However, it remains to be determined whether rabbit ADSCs (rADSCs) grown on the amniotic membrane can repair corneal epithelial defects in rabbits in vivo. We present the following article in accordance with the ARRIVE reporting checklist (available at http://dx.doi. org/10.21037/atm-20-4368).

\section{Methods}

Experiments were performed under a project license (No.: EENTIRB2018030101) granted by ethics board of China, in compliance with Chinese national or institutional guidelines for the care and use of animals.

\section{Materials and reagents}

\section{Materials}

Nine large white New Zealand rabbits (female, 2 to $2.5 \mathrm{~kg}$, Shanghai Institute of Zoology, Chinese Academy of Science) were included in the study. Adipose stem cells were isolated from subcutaneous adipose tissue at the back of the rabbits' necks.

\section{Reagents}

Collagenase Type I (Worthington Biochemical, USA), fetal bovine serum (HyClone, USA), DMEM powder (GIBCO, USA), L-glutamine (Shanghai Shisheng Biotechnology Co., Ltd.), ascorbic acid (Shanghai Shisheng Biotechnology Co., Ltd.), penicillin (North China Pharmaceutical Group), streptomycin (North China Pharmaceutical Group), trypsin (Shanghai Shisheng Biotechnology Co., Ltd.), dexamethasone (Sigma, USA), ascorbic acid (Sigma, USA), transferrin (Sigma, USA), NaHCO3 (Shanghai Hongguang Chemical Co.), monoclonal anti-vimentin antibody (LabVision, USA), monoclonal anti-smooth muscle actin antibody (LabVision, USA), $0.3 \%$ tobramycin/ $0.1 \%$ dexamethasone ophthalmic suspension (Alcon, USA), 0.4\% Benoxil (Santen Pharmaceutical, Japan), n-heptanol (Sinopharm Chemical Reagent Co., Ltd).

\section{Major instruments and supplies}

$5 \% \mathrm{CO}_{2}$ incubators at 37 degrees (Forma, USA), a disposable $10 \mathrm{~mL}$ syringe (Shanghai Misawa Medical Industry Co., Ltd.), Petri dishes, 24 and 6-well plate (Falcon, USA), centrifuge tubes (Falcon, USA), a centrifuge (Biofuge, Germany), low-temperature (refrigerated) highspeed centrifuges (Biofuge, Germany), an inverted phase contrast microscope (Olympus, Japan), precision balances (Mettler Toledo, Switzerland), an ultra-clean workbench (Shanghai Medical Device Company), adjustable volume pipettes (Eppendorf, USA), a vortex (Shanghai Medical Device Company), a thermostatic (temperature controlled) shaker (Taicang Huamei Biochemical Instrument Factory), an electrophoresis system (Tianneng Technology Co., Ltd., Shanghai), a thermal cycler (Biometra, USA), a laser scanning confocal microscope (Heidelberg, Germany), a s-520 scanning microscope (Hitachi, Japan), and a transmission electron microscope (Philips, Netherlands).

\section{Major culture media and reagents}

Dulbecco's Modified Eagle Medium (DMEM), 10\% fetal bovine serum (FBS), L-glutamine (300 mg/mL), ascorbic 
acid $(50 \mu \mathrm{g} / \mathrm{mL})$, penicillin $(100 \mathrm{U} / \mathrm{mL})$, and streptomycin $(100 \mathrm{U} / \mathrm{mL})$.

\section{Major surgical supplies}

Towel forceps, vessel forceps, scissors, needle holders, hooks, sterile towels, sterile gauzes, needles, 8-0 stitches, anesthetic and disinfecting supplies, microforceps, microscissors, and razor blades.

\section{Experimental methods}

\section{Cell isolation and culture}

To collect adipose tissue, the rabbits were anesthetized by intramuscular injection of $1 \mathrm{mg} / \mathrm{kg}$ ketamine and $0.5 \mathrm{mg} / \mathrm{kg}$ diazepam. The skin at the surgical site was prepared, disinfected, and submitted to local anesthesia. Two to three grams of subcutaneous adipose tissue at the back of the neck was cut and removed into sterile centrifuge tubes containing PBS under sterile conditions. The primary culture was completed in four hours. The surgical incision was stitched and locally treated with chlortetracycline ointment to prevent infection. The animals were returned to their cages after they woke. The adipose tissue was brought back to the lab for processing within two hours.

\section{Cell separation}

The rabbit adipose tissue was repeatedly rinsed with streptomycin solution and PBS, fully minced in a $10 \mathrm{~cm}^{2}$ petri dish, and then placed in a $50 \mathrm{~mL}$ centrifuge tube. Forty milliliters of $0.075 \%$ Type I collagenase solution was added to digest the tissue for $30 \mathrm{~min}$ on a 37-degree thermostatic shaker. Afterward, it was centrifuged at 1,500 rpm for $10 \mathrm{~min}$. After the supernatant was removed, the high-density cell pellet was obtained and was resuspended in DMEM with 10\% FBS.

\section{Seeding}

The cells were seeded in Petri dishes at a cell density of $10^{6} / \mathrm{mL}$, and the culture medium was added to a final volume of $8 \mathrm{~mL}$.

\section{Culture and passaging}

The cells were cultured at 37 degrees with $5 \% \mathrm{CO}_{2}$ and $100 \%$ saturated humidity for 48 to 72 hours before the medium was changed for the first time. A large number of adherent cells and floating blood cells were observed under an inverted phase contrast microscope. The floating cells were removed by repeated rinsing with PBS, and then DMEM with 10\% FBS was added. The next day, the culture medium was changed, and the cells were passaged when they reached $80 \%$ confluence. Before passaging, the cells were rinsed once with a small amount of PBS, and then two $\mathrm{ml}$ of $0.25 \%$ trypsin and $0.02 \%$ EDTA were added. Under a microscope, cytoplasmic shrinkage and morphologic rounding could be observed in most of the cells. Two milliliters of DMEM with serum was added to stop the trypsin digestion. The liquid was transferred into centrifuge tubes, which were then centrifuged at 1,500 rpm for $5 \mathrm{~min}$ to remove the supernatant. The tubes were gently tapped, and DMEM was added to resuspend cell pellet. The cell suspension was divided into three portions and seeded in culture dishes. The cells reached confluence after 3 to 4 days and were passaged again. rADSC cells from passage two were used for the experiments.

\section{Preparation of the amniotic membrane}

The amniotic membrane was provided by the Shanghai Research and Development Center of Tissue Engineering. Under sterile conditions, the amniotic membrane was immersed in DMEM for one hour. After the amniotic membrane was separated from the cellulose nitrate membrane filter, the amniotic membrane was laid flat on the culture dish using tweezers, and excess liquid was removed.

\section{Co-culturing of rADSCs and the amniotic membrane} Cultured rADSCs from passage two were rinsed once with a small amount of PBS and added to $2 \mathrm{~mL}$ of $0.25 \%$ trypsin and $0.02 \%$ EDTA. Cytoplasmic shrinkage and morphologic rounding could be observed in most cells. Two milliliters of DMEM with serum was added to stop the trypsin digestion, and the cell suspension was collected. The liquid was transferred into centrifuge tubes and centrifuged at 1,500 rpm for $5 \mathrm{~min}$ to remove the supernatant. The cells were resuspended and evenly dripped onto the amniotic membrane using pipettes. The membranes with rADSCs were kept in an incubator with $5 \% \mathrm{CO}_{2}$ and full humidity at 37 degrees for one hour. Then, $1 \mathrm{~mL}$ of DMEM was added, and the membrane was kept in the incubator for one more hour. Subsequently, $6 \mathrm{~mL}$ of DMEM was added, and the membranes with rADSCs were cultured in the incubator for a total of 7 days. The culture medium was changed every 2 days.

\section{Groups and the establishment of the rabbit LSCD model}

Nine healthy female adult New Zealand white rabbits weighing 2.0 to $2.5 \mathrm{~kg}$ were used to establish the model. The rabbits were randomly divided into three groups, and the left eyes were used for surgery. Control group 1 

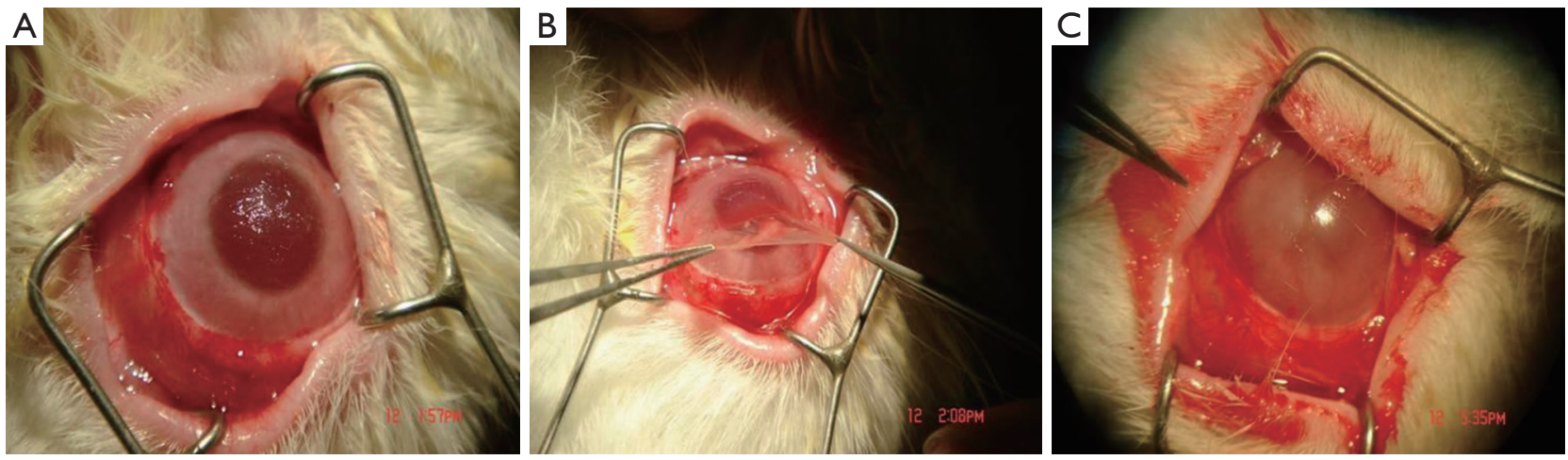

Figure 1 Surgical process. (A) The pannus is removed from the corneal surface; (B) the amniotic membrane is transplanted onto the corneal surface; (C) the membrane is sutured using 8-0 stitches.

comprised three rabbits treated with the LSCD model. Control group 2 comprised three rabbits that underwent amniotic membrane transplantation. The experimental group comprised three rabbits that underwent rADSC transplantation.

After the rabbits were anesthetized by intramuscular injection of $1 \mathrm{mg} / \mathrm{kg}$ ketamine and $0.5 \mathrm{mg} / \mathrm{kg}$ diazepam, Benoxil was dripped onto the surface of the cornea as an anesthetic to establish the LSCD model. Briefly, a cotton swab was dipped in n-heptanol and placed on the cornea. After approximately $1 \mathrm{~min}$, once the surface of the cornea appeared slightly turbid, the ocular surface was immediately rinsed with $200 \mathrm{~mL}$ of saline, and the corneal and limbal epithelia were scraped off. Three hundred sixty degrees of the surrounding superficial corneal limbal tissue was excised, including $1.5 \mathrm{~mm}$ of both the corneal and conjunctival anatomical junctions, respectively. After surgery, neomycin and dexamethasone ophthalmic was used twice daily for one week. The clinical manifestations of the LSCD model were observed daily until corneal opacity, neovascularization, and irregular epithelium were observed.

\section{Surgical procedure}

The operation was performed two weeks after the LSCD model was established. Anesthesia was induced using the method described above. Before surgery, fluorescent staining was performed to confirm that the epithelium had not grown. The pannus on the corneal surface was excised (Figure 1A). The conjunctiva was cut along the corneal limbus to expose the sclera, which is $4 \mathrm{~mm}$ behind the limbus. Subsequently, amniotic membrane with rADSCs or amniotic membrane only was sutured onto the cornea and sclera using 8-0 stitches with the cells facing up (Figure 1B).
The peripheral part of the amniotic membrane was covered with the free conjunctiva (Figure 1C). After a contact lens was fitted over the cornea and implanted, the palpebral fissure was sutured. After surgery, each rabbit was given neomycin and dexamethasone ophthalmic once per day for one week.

\section{Corneal score}

The suture was removed one week after surgery. Slit-lamp photographs were taken one, two, and four weeks after surgery, and the cornea was scored. The scoring criteria of the cornea were as follows: (I) corneal opacity: 0 points, the cornea is transparent with no turbidity; 1 point, the cornea is slightly cloudy, and iris texture is visible; 2 points, the cornea is moderately cloudy, and iris texture is unclear; 3 points, the cornea is severely cloudy, and the pupils are slightly visible; 4 points, the cornea is extremely cloudy with no visible pupil. (II) Sodium fluorescein staining of the corneal epithelium: 0 points, no corneal staining; 1 point, less than $1 / 4$ of the cornea is stained; 2 points, more than $1 / 4$ but less than $1 / 2$ of the cornea is stained; 3 points, more than $1 / 2$ but less than $3 / 4$ of the cornea is stained; 4 points, more than $3 / 4$ of the cornea is stained. (III) Corneal neovascularization: 0 points, no corneal neovascularization; 1 point, corneal neovascularization within $2 \mathrm{~mm}$ of the limbus; 2 points, corneal neovascularization around the cornea but in less than 2 quadrants; 3 points, corneal neovascularization is located around the cornea and in more than 2 quadrants; 4 points, neovascularization can be seen in the whole cornea. The data were analyzed using the statistical software Stata 7.0. The corneas in all groups were scored and analyzed using analysis of variance (ANOVA) at the same time point for a completely randomized design. 
In vivo dynamic observation of the repair of the corneal stroma using a confocal microscope

\section{Instrument and main parameters}

A laser confocal microscope (HRTIII/RCM) composed of the Heidelberg Retinal Tomography Laser Scanning System III (HRTIII, Germany) and the Rostock Corneal Module $(\mathrm{RCM})$ was used. The laser wavelength was $670 \mathrm{~nm}$, and the observation field was $380 \mu \mathrm{m} \times 380 \mu \mathrm{m}$ with $800 \times$ magnification. The horizontal and vertical resolutions were up to $1 \mu \mathrm{m}$.

\section{Operational procedure}

The eyes to be examined were topically anesthetized once with $0.4 \%$ benoxinate hydrochloride (Benoxil, Japan Santen Pharmaceutical Co., Ltd.) one month before and after surgery. The rabbits were positioned so that the treated eyes at the front of the operating table, with the mandible and forehead fixed on the mandibular support and forehead support, respectively. The depth of the focal plane of contact was preset to $0 \mu \mathrm{m}$ for both eyes. By changing the position of an indicator light, the cornea of the eyes to be inspected can be observed. After the examination, the images and videos were saved. The mandibular support and forehead support were cleaned using alcohol, and the disposable sterile corneal contact caps were replaced.

\section{Examination of the repair of corneal epithelium in each group by $\mathrm{H} \& \mathrm{E}$ staining}

One month after surgery, the rabbits were sacrificed, and the eyeballs were removed. After fixation, the eyes were embedded in paraffin following routine procedure, and the sections were cut to a thickness of $4 \gamma \mathrm{m}$. The sections were dewaxed using xylene and rehydrated through a series of ethanol gradients and a wash step using distilled water, as follows: xylene (I) for $5 \mathrm{~min}$, xylene (II) for $5 \mathrm{~min}, 100 \%$ ethanol for $2 \mathrm{~min}$, $95 \%$ ethanol for $1 \mathrm{~min}, 80 \%$ ethanol for $1 \mathrm{~min}, 75 \%$ ethanol for $1 \mathrm{~min}$, and distilled water for $2 \mathrm{~min}$. The sections were then dyed in hematoxylin for $5 \mathrm{~min}$ and rinsed with tap water. Subsequently, the sections underwent differentiation using acid alcohol solution for 30 seconds (the sections were lifted out of and put back into the solution several times) and then immersed in tap water or warm water (approximately 50 degrees) for $5 \mathrm{~min}$. Then, the sections were dyed in the eosin solution for $2 \mathrm{~min}$. The routine procedure of dehydration, clearing, and sealing was performed as follows: $95 \%$ ethanol (I) for $1 \mathrm{~min}, 95 \%$ ethanol (II) for $1 \mathrm{~min}, 100 \%$ ethanol (I) for $1 \mathrm{~min}, 100 \%$ ethanol (II) for $1 \mathrm{~min}$, xylene carbonate (3:1) for $1 \mathrm{~min}$, xylene (I) for $1 \mathrm{~min}$, xylene (II) for $1 \mathrm{~min}$, and sealing using neutral resin. Pictures were then taken.

\section{Detection of K3/K12 (cornea-specific keratins) expression by immunohistochemical staining} Paraffin slides were cut from the wax blocks used for H\&E staining. (I) The paraffin sections were dewaxed using the following routine procedure: immersion in xylene three times for $10 \mathrm{~min}$ each to dissolve paraffin; treated with $100 \%$ ethanol, $95 \%$ ethanol, $80 \%$ ethanol and tap water for $3 \mathrm{~min}$ each, and then rinsed with PBS three times of 3 min each. (II) The sections were incubated in $3 \% \mathrm{H}_{2} \mathrm{O}_{2}$ for $30 \mathrm{~min}$ at room temperature to block endogenous peroxidase. (III) The samples were rinsed with PBS 3 times for 3 min each. (IV) They were then incubated in $1 \%$ trypsin at 37 degrees for $30 \mathrm{~min},(\mathrm{~V})$ rinsed with PBS three times for 3 min each, and (VI) blocked using serum at 37 degrees for $30 \mathrm{~min}$, and then the residual liquid was removed. (VII) Primary antibodies against K3 and K12 were added, and the samples were incubated at 4 degrees overnight. (VIII) The samples were rinsed with PBS three times for 5 min each. (IX) Biotin-conjugated secondary antibodies were added, and the samples were incubated at 37 degrees for $30 \mathrm{~min}$ and then rinsed with PBS three times for 5 min each. (X) Freshly prepared diaminobenzidine (DAB) solution was added to visualize the staining, and the results were observed using a microscope. Yellow-brown staining in the cytoplasm was considered positive staining. (XI) The sections were counterstained using hematoxylin, dehydrated, cleared, and sealed for photography.

Scanning electron microscopy and transmission microscopy were carried out at the electron microscopy facility of Shanghai Medical College Fudan University.

\section{Results}

\section{Establishment of the rabbit LSCD model (Figure 2)}

Two weeks after surgery, it was observed that corneal epithelial cells peeled off together, presented stromal edema and were opaque; iris texture was unclear, the pupils were hardly visible, and limbal neovascularization had developed (Figure 2A). Fluorescein eye staining showed a large area of corneal epithelial defects and positive staining (Figure 2E). After three weeks, the corneal stromal opacity and edema were alleviated, but the area of the corneal epithelial defect was still large (Figure 2B,F). After four weeks, new blood vessels gradually grew into the pupil area (Figure $2 C, G$ ). 

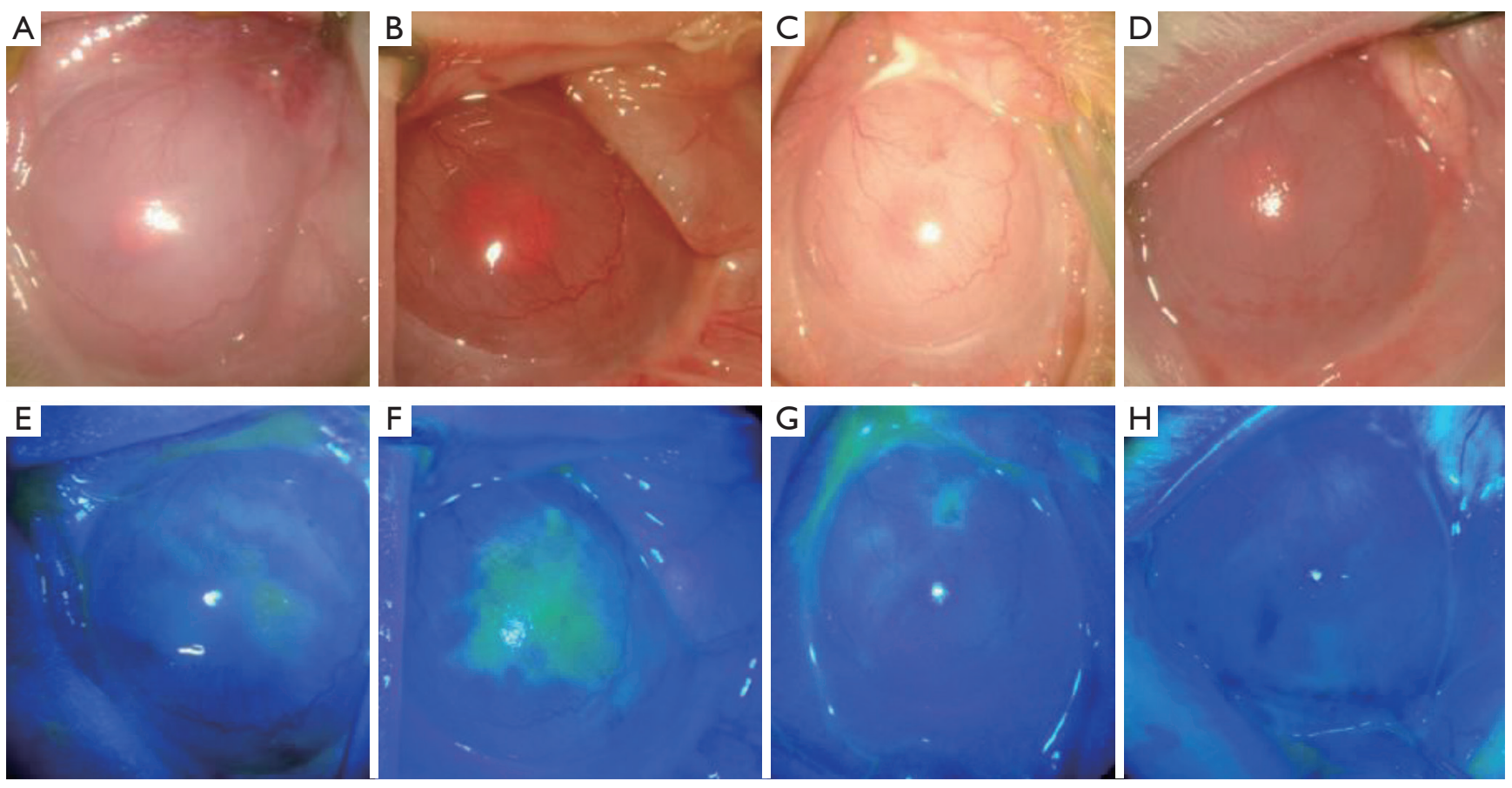

Figure 2 Control group 1: limbal stem cell defect model. (A and E) Preoperative corneal opacity, neovascularization, and a large area of staining in the corneal epithelium; (B and F) one week after surgery; (C and G) two weeks after surgery; (D and H) one month after surgery. There was no significant improvement in corneal opacity, and new blood vessels gradually grew into the pupil area.

After six weeks, a large area of the corneal epithelium was still defective, and neovascularization could be observed throughout the whole cornea (Figure 2D,H).

\section{Changes in the rabbit cornea after amniotic membrane transplantation (Figure 3)}

Amniotic membrane transplantation was performed two weeks after the LSCD model was established (Figure 3A,E). The stitches at the eyelid margin were removed one week after surgery. There was no significant improvement in corneal transparency one and two weeks after surgery compared with the previous condition, and part of the corneal epithelium still had defects and showed positive fluorescein staining. In addition, new blood vessels gradually grew from the corneal limbus into the pupil area (Figure 3B,C,F,G). One month after surgery, epithelial edema was slightly alleviated compared with the previous condition, and epithelial defects were reduced. However, there were no clear differences in corneal transparency and neovascularization compared with the condition before surgery (Figure 3D,H).

\section{Changes in the rabbit cornea after the rADSC transplantation (Figure 4)}

Autologous rADSC transplantation was also performed two weeks after the LSCD model was established (Figure 4A,E). One week after surgery, the stitches were removed. The cornea was edematous and cloudy, the pupil was invisible, and half of the corneal field had neovascularization. In addition, a large area of the central cornea had defects and positive fluorescein staining (Figure 4B,F). Two weeks after surgery, the corneal edema was alleviated, and the pupil was visible. Furthermore, the number of new blood vessels was reduced compared with the condition in the previous week (Figure $4 C, G$ ). Four weeks after surgery, the cornea was significantly more transparent than it had been before surgery, and the neovascularization was reduced compared with the previous period. Only a small portion of the cornea had positive fluorescein staining (Figure 4D,H).

\section{Cornea scores}

The corneas of the nine rabbits that underwent surgery were scored (Table 1). All LSCD models were successfully 

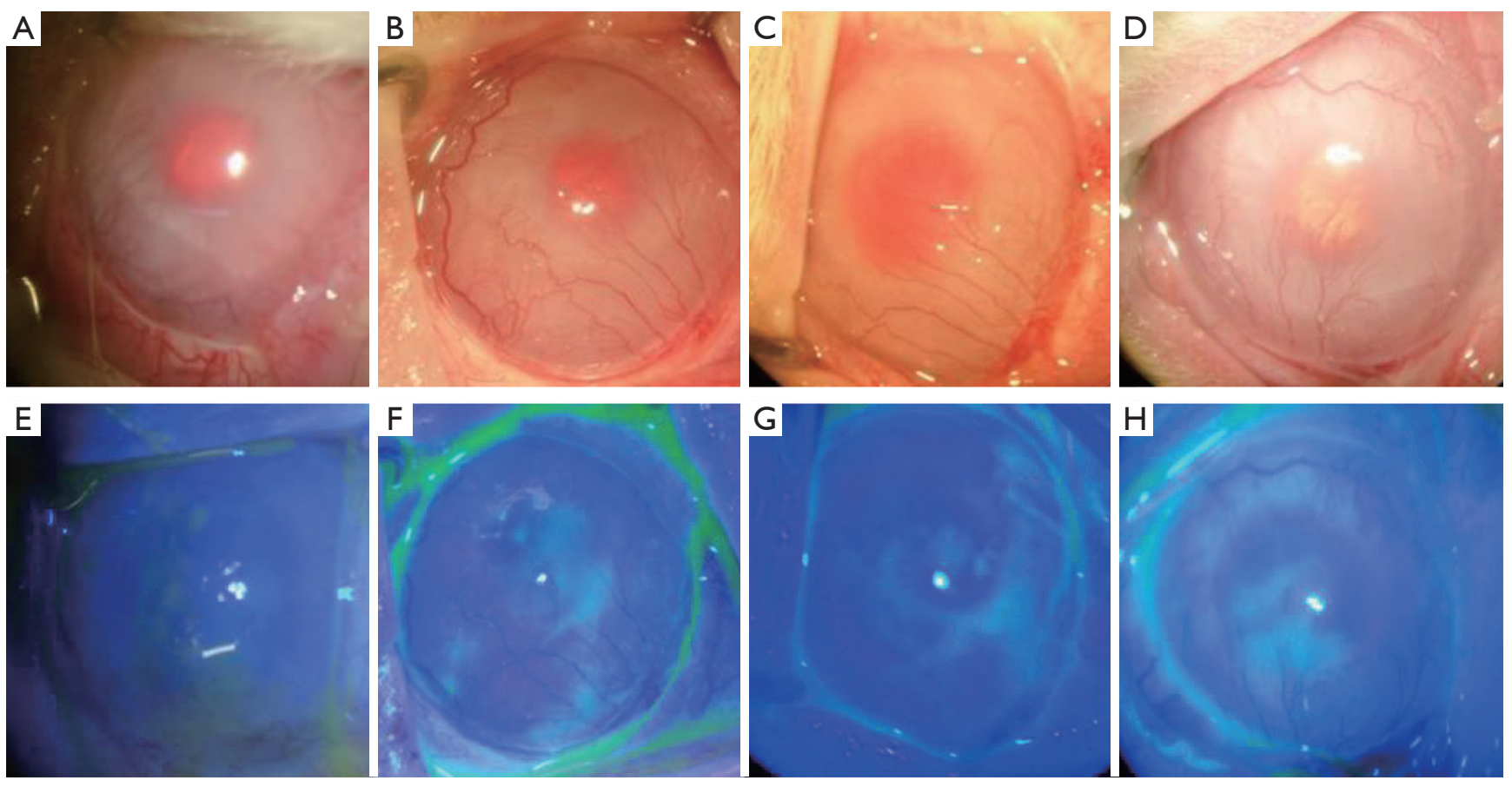

Figure 3 Control group 2: the amniotic membrane transplantation group. (A and E) Corneal opacity, neovascularization, and positive sodium fluorescein staining before surgery; (B and F) one week after surgery; (C and G) two weeks after surgery; (D and H) one month after surgery. Corneal epithelial edema was slightly alleviated compared with the previous conditions, and epithelial defects were reduced. However, there was no apparent difference in corneal transparency and neovascularization compared with before surgery.

established before surgery. There was no significant difference among the scores of all groups $(\mathrm{F}=4.45$, $\mathrm{P}=0.07,>0.05)$. No significant differences were observed between the defect group and the amniotic membrane transplantation group at two weeks $(\mathrm{F}=-2.33, \mathrm{P}=0.248$, $>0.05)$ and four weeks $(\mathrm{F}=-2.67, \mathrm{P}=0.432,>0.05)$ after surgery. Two weeks after surgery, the scores of the corneas in the rADSC combined with amniotic membrane transplantation group were lower than those of the corneas in the defect group $(\mathrm{F}=-7, \mathrm{P}=0.002,<0.05)$ and the amniotic membrane transplantation group $(\mathrm{F}=-4.67, \mathrm{P}=0.018,<0.05)$, with significant differences. Four weeks after surgery, the scores of the corneas in the rADSC combined with amniotic membrane transplantation group were lower than those of the corneas in the defect group $(\mathrm{F}=-8, \mathrm{P}=0.007$, $<0.05)$ and the amniotic membrane group $(\mathrm{F}=-5.33$, $\mathrm{P}=0.046,<0.05$ ), with significant differences. There was a significant difference in the scores of the corneas in the rADSC combined with amniotic membrane transplantation group between two weeks after surgery and before surgery $(\mathrm{F}=-4.67, \mathrm{P}=0.009,<0.05)$. A significant difference was also present between the scores at four weeks after surgery and before surgery ( $\mathrm{F}=-5, \mathrm{P}=0.007,<0.05)$.

\section{In vivo dynamic assessment of the corneal epithelium in each experimental group using corneal confocal microscopy (CM) observation four weeks after surgery}

\section{Normal corneal epithelium}

Confocal analysis showed that normal superficial epithelial cells appeared to have an orderly, polygonal arrangement and had low cytoplasmic reflection, a large nuclear volume, high reflection, and unclear cell boundaries (Figure $5 A$ ).

\section{The corneal epithelium of the LSCD group}

Confocal analysis showed a large defect area in the corneal epithelium; directly visible, high-brightness, strip-like bars in the active state; and corneal stromal cells. However, sporadic corneal epithelial cells could still be observed (Figure 5B).

\section{The corneal epithelium of the amniotic membrane transplantation group}

Confocal analysis showed that cells had different sizes 

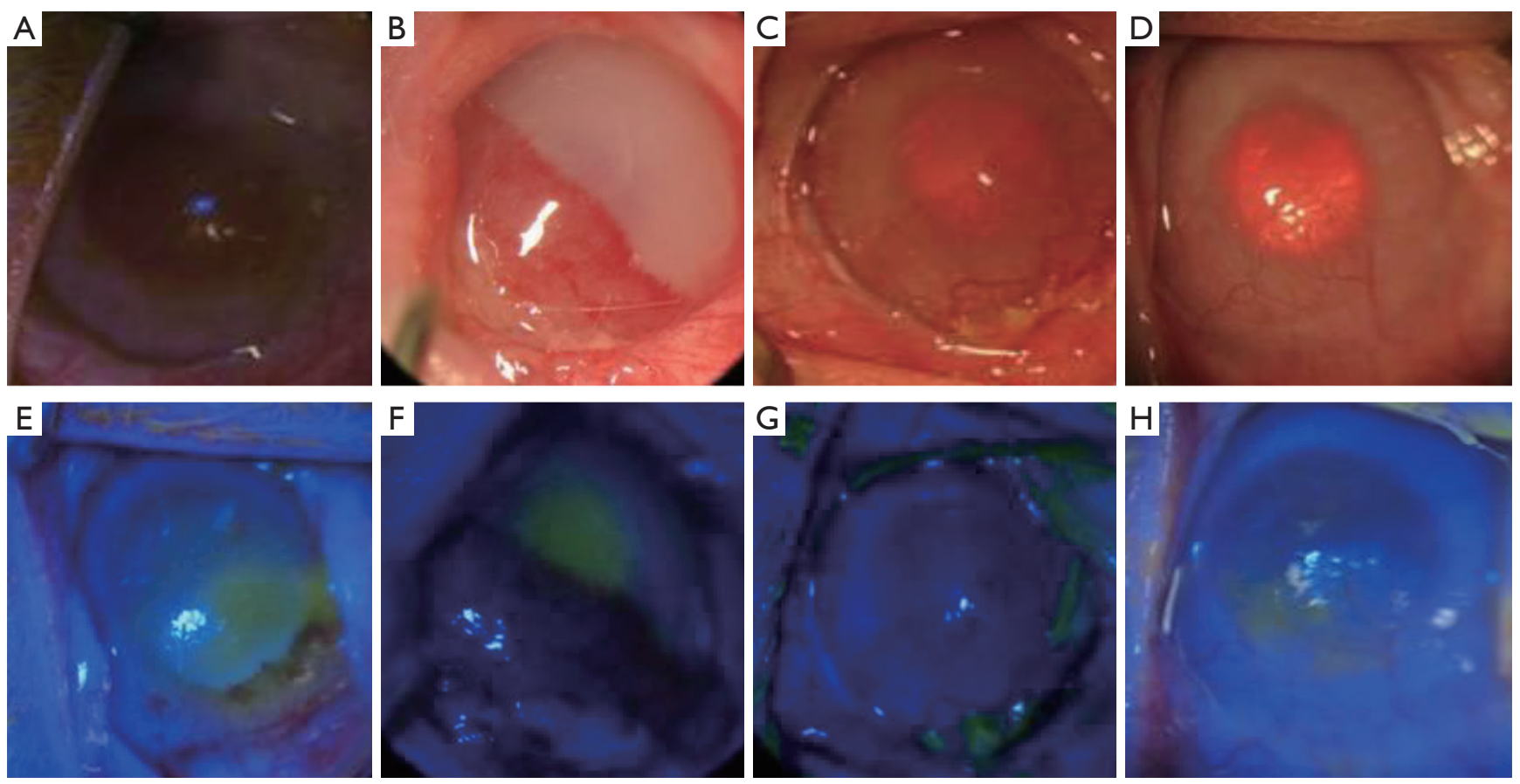

Figure 4 The experimental group: the rADSCs transplantation group. (A and E) The cornea was cloudy before surgery and was infiltrated with new blood vessels. The epithelium was deficient in fluorescent staining. (B and F) One week after surgery, the cornea had edema and was cloudy. The pupils could not be seen, and there were a large number of new blood vessels. (C and G) The cornea gradually became transparent, and neovascularization was not significantly improved compared with the previous condition. (D and H) The cornea was transparent. The number of new blood vessels was obviously reduced. Fluorescent staining revealed small areas of defects in the epithelium. rADSC, rabbit adipose tissue-derived stem cell.

Table 1 Scores for the rabbit corneas in each group

\begin{tabular}{lccc}
\hline Group & Before surgery & 2 weeks after surgery & 4 weeks after surgery \\
\hline LSCD group & 6 & 8 & 8 \\
& 6 & 10 & 10 \\
Amniotic membrane transplantation group & 7 & 7 & 6 \\
& 9 & 7 & 9 \\
rADSCs group & 6 & 8 & 3 \\
& 7 & 3 & 5 \\
\hline
\end{tabular}

LSCD, limbal stem cell deficiency; rADSC, rabbit adipose tissue-derived stem cell. 

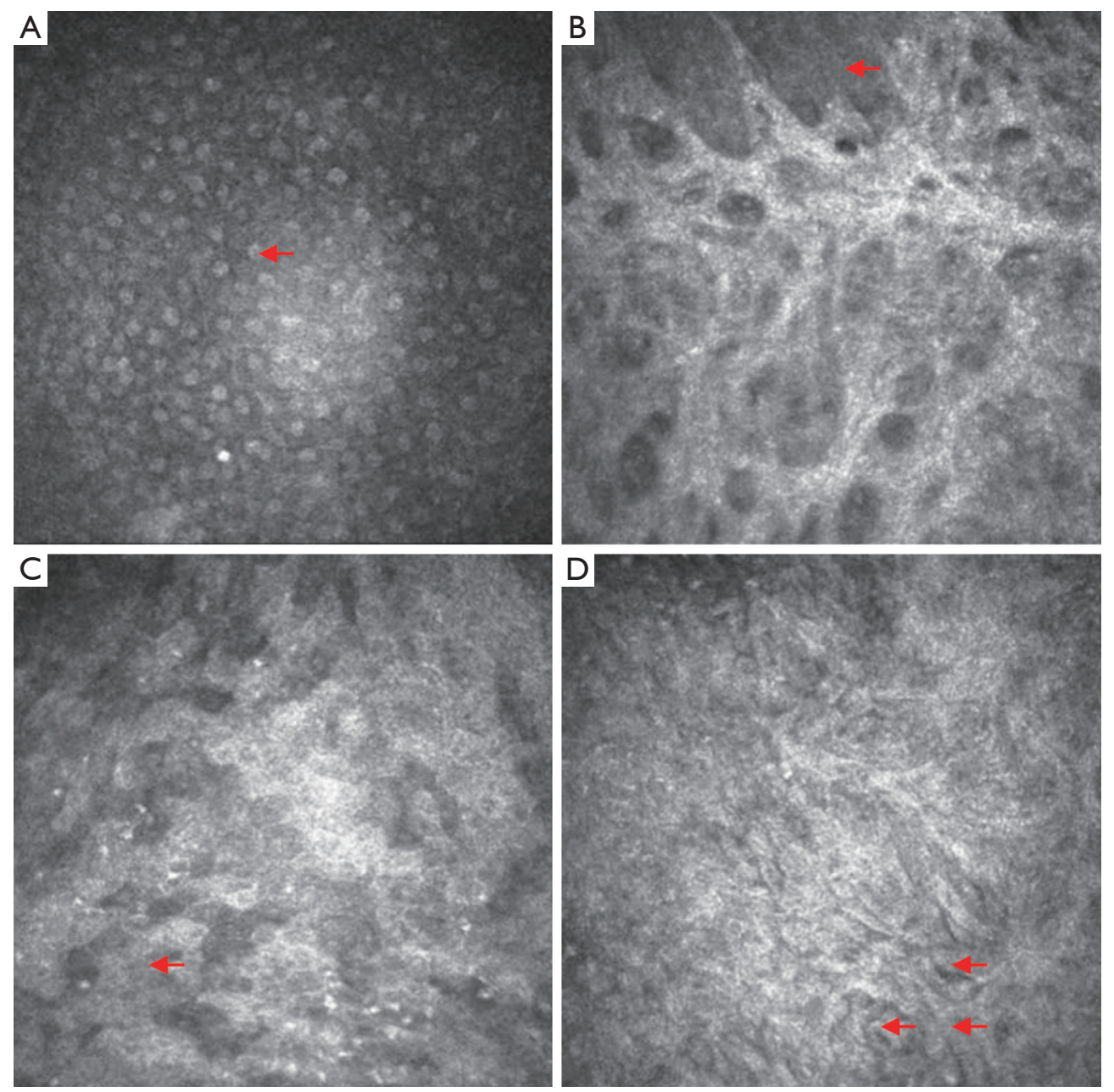

Figure 5 In vivo dynamic observation of the corneal epithelium using confocal corneal microscopy. (A) Normal corneal epithelium. The red arrows point to the cornea, with low-reflection cytoplasm and a high-reflection nucleus. (B) Corneal epithelial defects. The exposed collagen matrix layer can be directly observed. The red arrow points to the remaining corneal epithelial cells. (C) Example from the amniotic membrane transplantation group. A large number of irregular cells can be seen. The arrow points to inflammatory cells. (D) Example from the rADSC combined with amniotic membrane transplantation group. The arrows point to spindle-shaped, tightly arranged cells. rADSC, rabbit adipose tissue-derived stem cell.

and irregular shapes and were unevenly distributed. The epithelium was infiltrated by inflammatory cells, and there were cell-free dark regions (Figure 5C).

\section{The corneal epithelium in the rADSC combined with amniotic membrane transplantation group \\ Confocal analysis showed that spindle-shaped cells with unevenly distributed, high-brightness and reflective cytoplasm and low-reflective nuclei were tightly arranged. Round cells with a homogeneous nucleus could still be observed in the surrounding area (Figure 5D).}

\section{Evaluation of corneal epithelial repair according to $H \& E$ staining}

\section{Normal control group}

There were four or five layers in the corneal epithelium, which had a regular arrangement. Collagen fibers in the stromal layer were neatly arranged. No infiltration of inflammatory cells and neovascularization could be observed (Figure 6A).

\section{The LSCD group}

Corneal epithelial cells were absent. Corneal stromal edema 

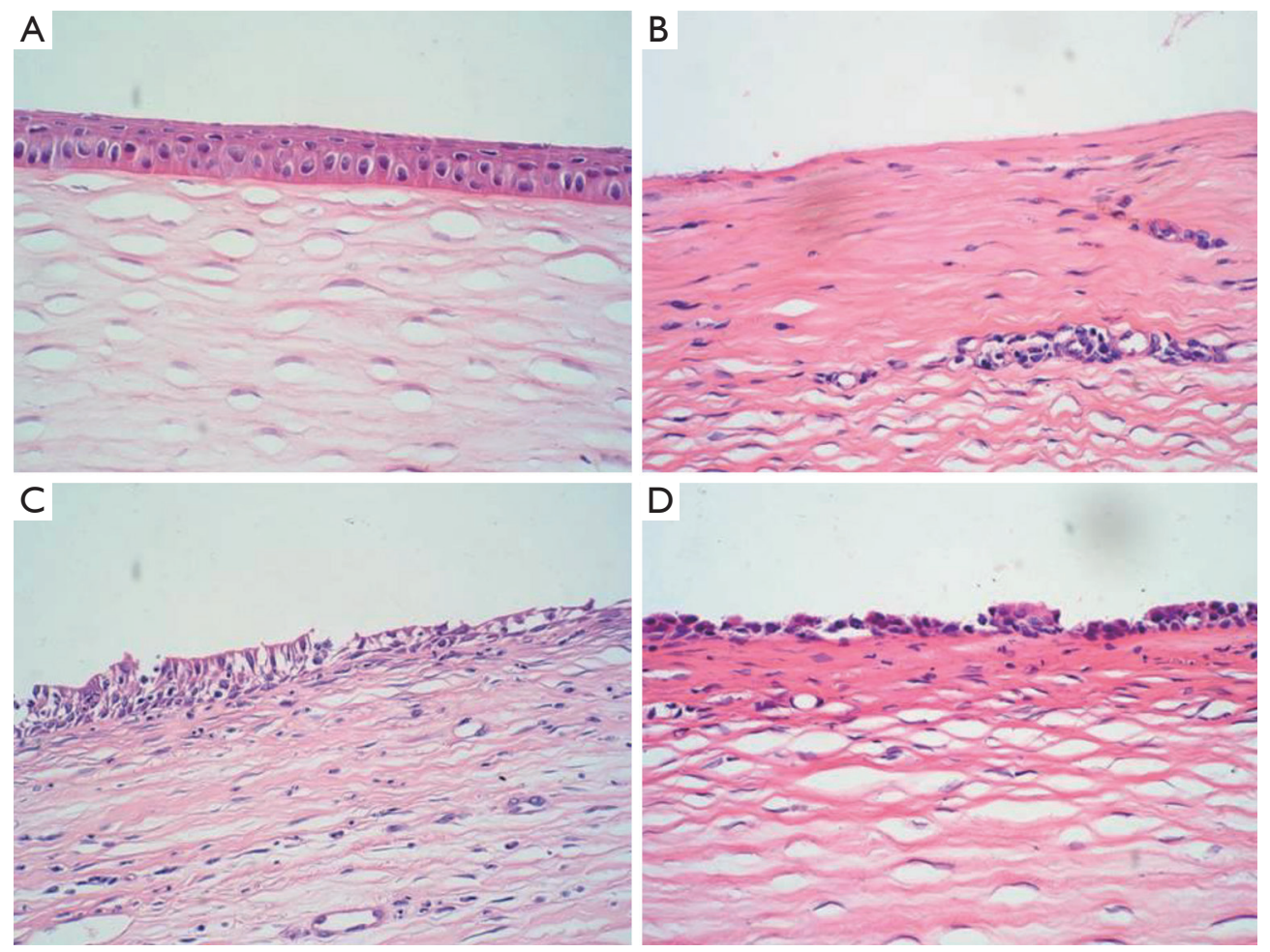

Figure 6 Assessment of corneal epithelium repair by H\&E staining ( $\times 100)$. (A) Normal corneal epithelium; (B) corneal epithelial defect. There was infiltration of inflammatory cells; (C) loose cells in the superficial layer. A large number of inflammatory cells were present; (D) cells arranged in one or two layers on the surface.

was obvious. In the stromal layer, there were infiltrated inflammatory cells, collagen fibers had a disorderly arrangement, and neovascularization could be observed (Figure 6B).

\section{The amniotic membrane transplantation group}

Two or three layers of the corneal epithelium were present, but their arrangement was loose, disordered, and irregular in shape. The stromal layer had a moderate number of inflammatory cells, and stromal edema was milder. Sparse new blood vessels could be observed in the superficial stromal layer, and the lumen was thin (Figure 6C).

\section{The rADSC combined with amniotic membrane transplantation group}

One or two layers of epithelial cells could be observed on the surface of the cornea. The cell arrangement had no polarity. However, it was difficult to determine the phenotype using routine pathological examination. No goblet cells were found. A small number of inflammatory cells infiltrated the stroma layer, and collagen fibers were arranged relatively neatly (Figure 6D).

\section{Evaluation of the expression of the corneal epithelium- specific marker K3/K12 using immunobistochemical staining}

$\mathrm{K} 3 / \mathrm{K} 12$ was expressed in the normal corneal epithelium group (Figure $7 A$ ) but not in the defect group (Figure $7 B$ ). There were a few sporadic positive signals in the amniotic membrane transplantation group (Figure 7C). A small number of cells in the rADSC combined with amniotic membrane transplantation group had positive expression (Figure 7D).

\section{Evaluation of the corneal epithelial cells in each group using scanning electron microscopy}

\section{Normal control group}

The superficial corneal epithelial cells had a polygonal shape and were neatly arranged. The cell boundaries were clear, and there were two or more renewing pore-like 

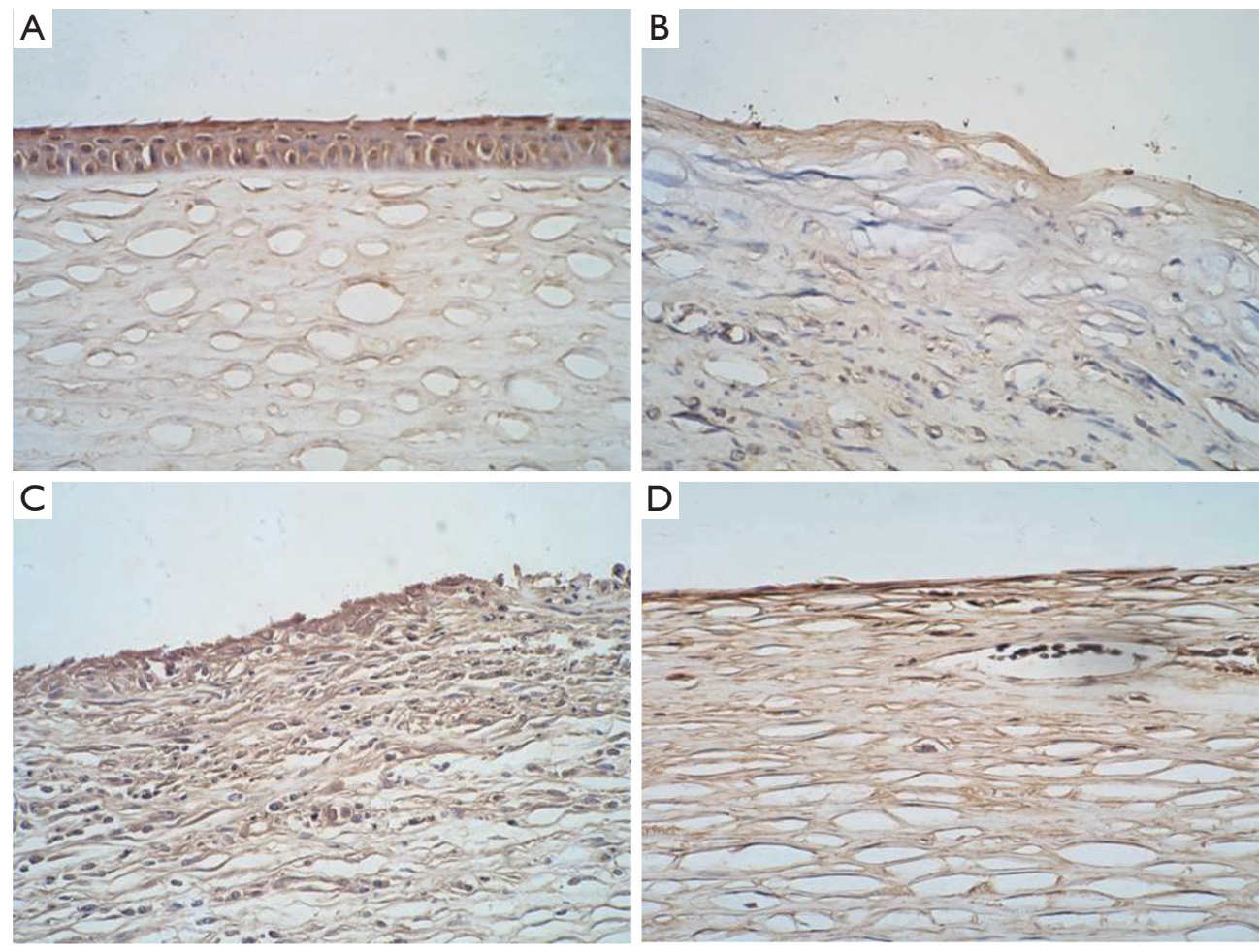

Figure 7 Detection of K3/K12 expression using immunohistochemistry ( $\times 100)$. (A) Normal corneal epithelial cells with K3/K12 expression; (B) corneal epithelial defect; (C) the amniotic membrane transplantation group. A small number of cells on the surface had positive expression; (D) the rADSCs transplantation group. K3/K12 expression was negative. rADSC, rabbit adipose tissue-derived stem cell.

openings. Furthermore, the binding between the cells was tight and without gaps (Figure $8 A$ ).

\section{The LSCD group}

The corneal epithelium had fallen off. Collagen fibers could be observed in the corneal stroma layer, and the collagen fiber bundles were parallel to each other and closely stacked in layers (Figure 8B).

\section{The amniotic membrane transplantation group}

A few epithelial cells with irregular morphology could be observed on the surface. Most of the renewing pore-like openings had disappeared, and cell activity was decreased. There were large gaps between cells, and most of the cells had fallen off (Figure 8C).

\section{The rADSC group}

Spindle-shaped cells could be observed on the surface and were higher than the surface level. They were surrounded by cells with different shapes and sizes that were tightly connected (Figure 8D).

\section{Evaluation of the corneal epithelial cells in each group using transmission electron microscopy}

\section{Normal control group}

The structures of the organelles and the nuclei in the corneal epithelial cells were normal. The structure of the basement membrane was intact. The connection between the cells in the corneal basal layer via desmosomes was clearly visible. The epithelial cells were bound to the stroma very tightly (Figure 9A).

\section{The LSCD group}

In the corneal epithelial cells, the cell membrane had dissolved, and the nucleus had disintegrated. The gap between the cells had increased. The shape of the cells was irregular, and the cells were arranged loosely. The connection via hemidesmosomes between epithelial cells and the basement membrane had disappeared; therefore, the connection with the matrix was not tight (Figure 9B).

\section{The amniotic membrane transplantation group}

In most areas, the epithelial cells had lost their normal 

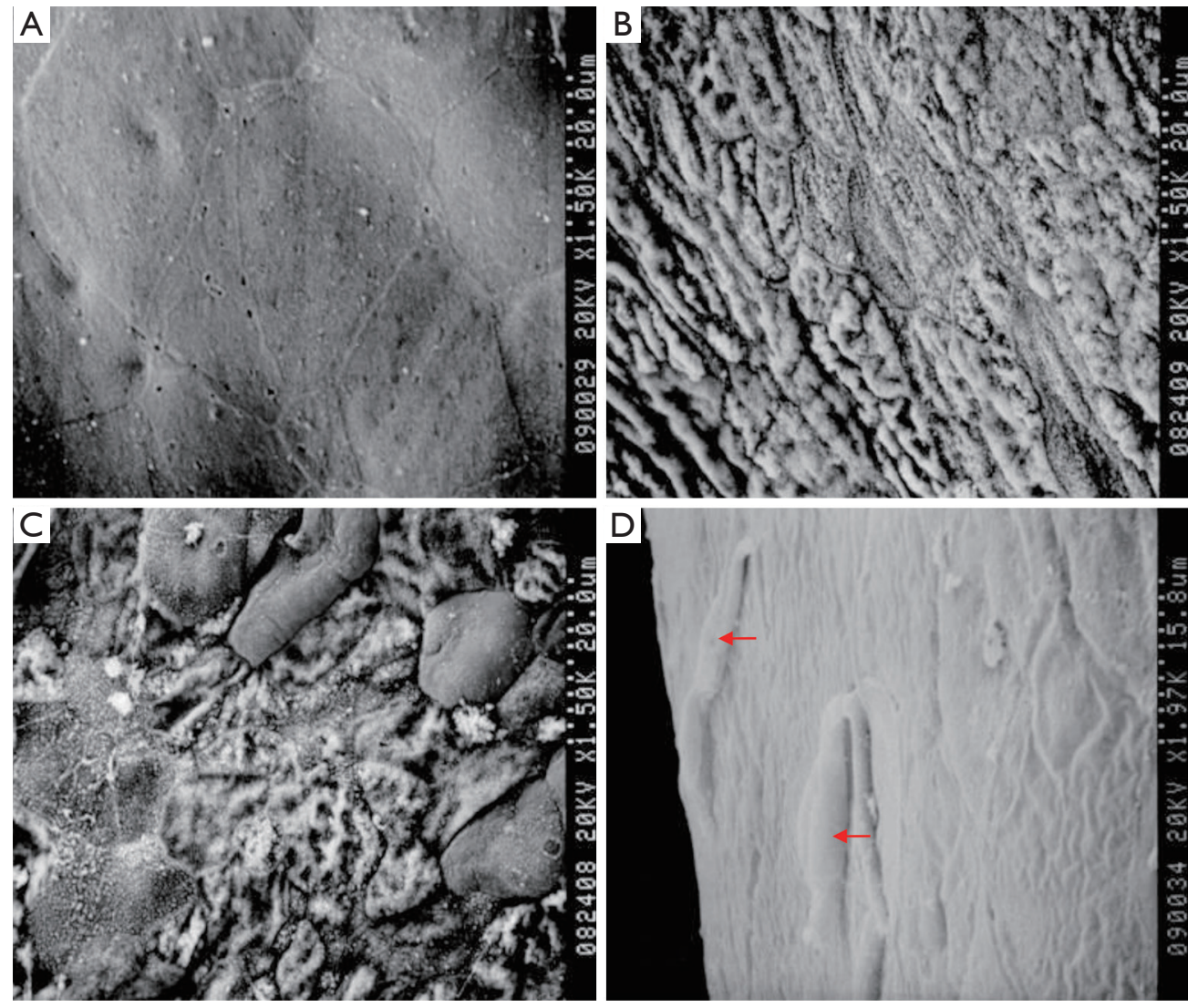

Figure 8 Observation of the corneal surface using scanning electron microscopy. (A) normal corneal epithelium; (B) the LSCD group. Only exposed collagen fibers are visible; (C) the amniotic membrane transplantation group; (D) the rADSCs transplantation group. Spindleshaped cells are present (indicated by arrows). LSCD, limbal stem cell deficiency; rADSC, rabbit adipose tissue-derived stem cell.

polarity. The cell layers were destroyed, and the cells were only separated into one to three layers. The gap between the cells was relatively large. The organelles were sparse and disorderly. The structure of the front elastic layer had disappeared (Figure 9C).

\section{The rADSC group}

Relatively larger cells with intact organelles could be observed in the corneal epithelium. The cells were obviously different from the corneal epithelial cells (Figure 9D).

\section{Discussion}

In clinical practice, many serious ocular surface disorders, such as severe trauma (chemical injuries, thermal burns, etc.), Stevens-Johnson syndrome, and ocular cicatricial pemphigoid, are related to LSCD. Eventually, all these diseases will lead to blindness because the corneal epithelium loses the ability to repair and regenerate. At present, allogeneic corneal transplantation or limbal transplantation is the most common method for corneal surface reconstruction; however, due to the shortage of organ donors, many patients face treatment delays.

In vitro cultured ADSCs can be induced to differentiate into mature adipocytes, osteocytes, neurocytes, and endothelial cells under specific conditions. Furthermore, they can differentiate into different germ layers under specific culture conditions (3-7). This ability offers the possibility of rebuilding the ocular surface using adipose stem cells. The amniotic basement membrane is degradable and can promote adherent cells to grow and proliferate at the same time; therefore, it is an ideal carrier for use on the ocular surface. The first part of the study showed that adipose stem cells grow well on the surface of the amniotic membrane and maintain a good morphology. The data 

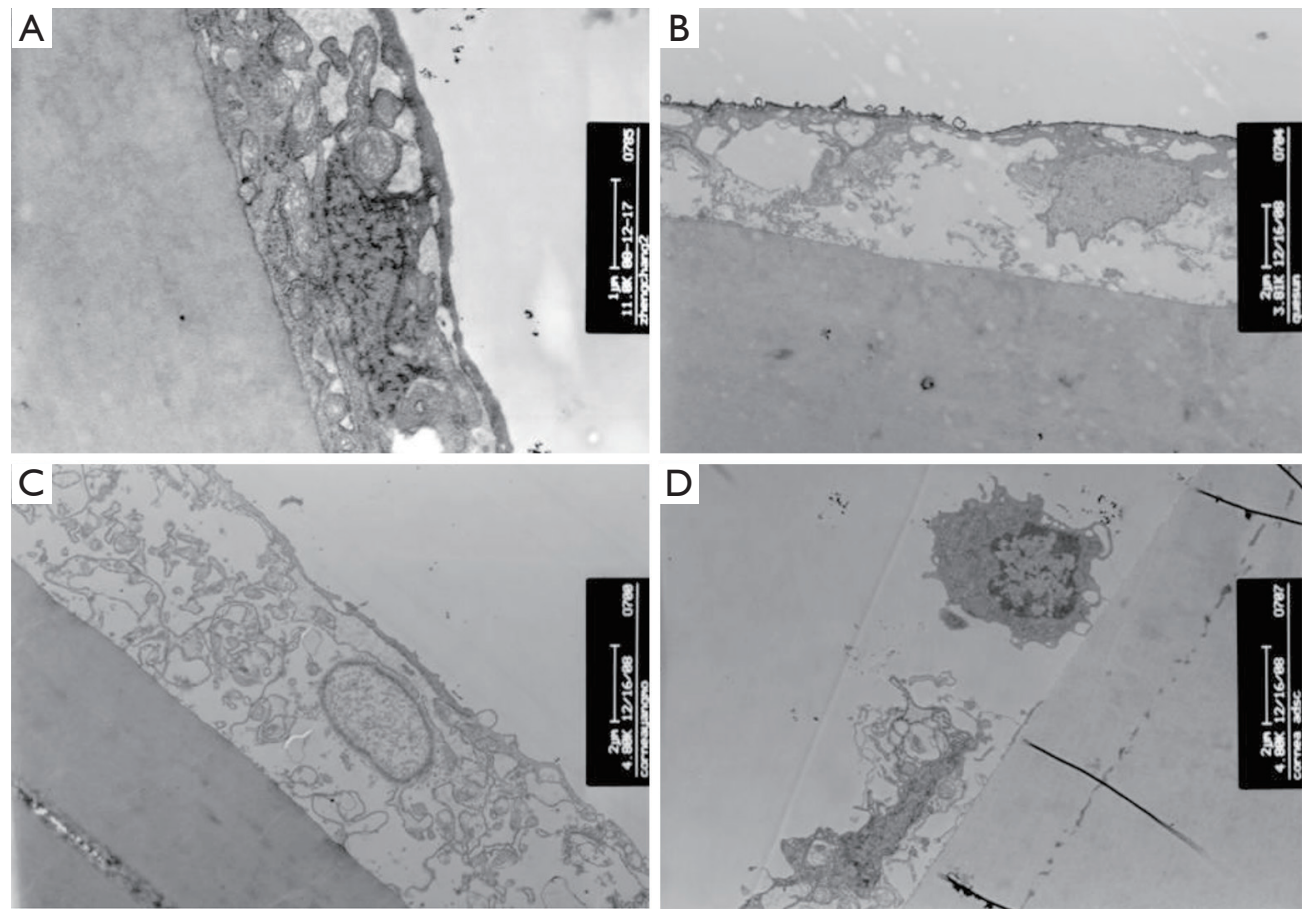

Figure 9 Observation of the connection between corneal epithelial cells and the stroma layer using transmission electron microscopy. (A) Normal corneal epithelium; (B) the LSCD group; (C) the amniotic membrane transplantation group; (D) the rADSCs group. LSCD, limbal stem cell deficiency; rADSC, rabbit adipose tissue-derived stem cell.

established a theoretical and experimental foundation for the next-stage animal testing of tissue engineering for corneal epithelial transplantation.

The LSCD model is generated using chemical injury and removal of the limbal tissue. The reason $n$-heptanol was used instead of acid/alkali was mainly because we did not want to damage the corneal stroma and intended to preserve the corneal stromal microenvironment to allow better observation of whether adipose stem cells can be induced to differentiate into corneal epithelial cells.

In this study, we designed an experimental rADSC combined with amniotic membrane transplantation group and two control groups-the LSCD group and the amniotic membrane transplantation group-to examine corneal self-repair after limbal injury and the effect of the amniotic membrane on corneal epithelial reconstruction. By scoring the cornea using a slit lamp examination and fluorescent staining, we found that the efficacy of rADSC transplantation for treating LSCD and reconstructing the epithelium was significantly better than that of the other two methods. Furthermore, the cornea was epithelialized with increased transparency two weeks after surgery, and the area of neovascularization was smaller than before surgery. However, H\&E staining revealed that the rADSCs formed fewer layers on the corneal surface than are present in normal corneal epithelium. In vivo CM showed that spindle-shaped cells with unevenly distributed, high-brightness and high-reflection cytoplasm and lowreflection nuclei were tightly arranged, and round cells with homogeneous nuclei could still be seen in the surrounding area one month after rADSC transplantation. Scanning transmission electron microscopy observation showed that spindle-shaped cells were distributed on the surface of the cornea. Immunohistochemical staining showed that after transplantation, the cornea did not express the corneal epithelial cell marker K3/K12, suggesting that rADSCs do not differentiate into corneal epithelial cells. However, given the lack of longer-term observation and cytokine detection, further study is needed to determine why the cornea becomes transparent. There are studies suggesting that adult stem cells can secrete factors that inhibit neovascularization and inflammation (8).

The use of autologous ADSCs on amniotic membrane can avoid rejection reactions. There are no ethical and 
moral issues, and supplies are ample. Furthermore, our experiments have demonstrated that they have good efficacy for treating LSCD. However, because our observation time was short, the long-term effects and underlying mechanism of this method remain to be explored. In conclusion, autologous ADSCs and amniotic membrane provide potential new options for ocular surface reconstruction and are a promising tissue engineering strategy.

\section{Acknowledgments}

Funding: None.

\section{Footnote}

Reporting Checklist: The authors have completed the ARRIVE reporting checklist. Available at http://dx.doi. org/10.21037/atm-20-4368

Data Sharing Statement: Available at http://dx.doi. org/10.21037/atm-20-4368

Conflicts of Interest: All authors have completed the ICMJE uniform disclosure form (available at http://dx.doi. org/10.21037/atm-20-4368). The authors have no conflicts of interest to declare.

Ethical Statement: The authors are accountable for all aspects of the work in ensuring that questions related to the accuracy or integrity of any part of the work are appropriately investigated and resolved. The study was approved by the Ethics Committee of Eye, Ear, Nose, and Throat Hospital of Fudan University. Experiments were performed under a project license (No.: EENTIRB2018030101) granted by ethics board of China, in compliance with Chinese national or institutional guidelines for the care and use of animals.

Open Access Statement: This is an Open Access article distributed in accordance with the Creative Commons

Cite this article as: Qiu T, Cui L, Xu JJ, Hong JX, Xiang J. Reconstruction of the ocular surface by autologous transplantation of rabbit adipose tissue-derived stem cells on amniotic membrane. Ann Transl Med 2020;8(17):1062. doi: 10.21037/atm-20-4368
Attribution-NonCommercial-NoDerivs 4.0 International License (CC BY-NC-ND 4.0), which permits the noncommercial replication and distribution of the article with the strict proviso that no changes or edits are made and the original work is properly cited (including links to both the formal publication through the relevant DOI and the license). See: https://creativecommons.org/licenses/by-nc-nd/4.0/.

\section{References}

1. Lamm V, Hara H, Mammen A, et al. Corneal blindness and xenotransplantation. Xenotransplantation 2014;21:99-114.

2. Hong J, Xu J, Li G, et al. Proliferation capacity of rabbit adipose tissue-derived mesenchymal stem cells at early stage in vitro. XXX 2008;12:10077-80.

3. Waldner M, Zhang W, James IB, et al. Characteristics and immunomodulating functions of adipose-derived and bone marrow-derived mesenchymal stem cells across defined human leukocyte antigen barriers. Front Immunol 2018;9:1642.

4. Si Z, Wang X, Sun C, et al. Adipose-derived stem cells: Sources, potency, and implications for regenerative therapies. Biomed Pharmacother 2019;114:108765.

5. Garcia-Honduvilla N, Cifuentes A, Ortega MA, et al. High Sensitivity of Human Adipose Stem Cells to Differentiate into Myofibroblasts in the Presence of C. aspersa Egg Extract. Stem Cells Int 2017:9142493.

6. Jung YJ, Park YY, Huh JW, et al. The effect of human adipose-derived stem cells on lipopolysaccharide-induced acute respiratory distress syndrome in mice. Ann Transl Med 2019;7:674.

7. Kasir R, Vernekar VN, Laurencin CT. Regenerative engineering of cartilage using adipose-derived stem cells. Regen Eng Transl Med 2015;1:42-9.

8. Avila M, España M, Moreno C, et al. Reconstruction of Ocular Surface With Heterologous Limbal Epithelium and Amniotic Membrane in a Rabbit Model. Cornea 2001;20:414-20. 